\title{
ADMISSIBLE BOUNDARY VALUES OF BOUNDED HOLOMORPHIC FUNCTIONS IN WEDGES
}

\author{
FRANC FORSTNERIC
}

\begin{abstract}
If $M \subset \mathbb{C}^{N}$ is a generic Cauchy-Riemann manifold and $\mathscr{W} \subset \mathbb{C}^{N}$ is a wedge domain with edge $M$, then every bounded holomorphic function on $\mathscr{W}$ has an admissible limit at almost every point of $M$. Moreover, if a holomorphic function $f$ on $\mathscr{W}$ has a distribution boundary value (bv $f$ ) on $M$ that is a bounded measurable function, then $f$ is bounded on every finer wedge near $M$, and its admissible limit equals (bv $f)(p)$ at almost every point $p \in M$.
\end{abstract}

\section{INTRODUCTION}

In this article we prove the existence of pointwise boundary values of bounded holomorphic functions in wedge domains with generic edges (Theorem 1). We also prove a more technical result concerning the boundary values of nonholomorphic functions in a standard wedge with edge $\mathbb{R}^{N} \times \mathbb{R}^{l} \subset \mathbb{R}^{N} \times \mathbb{C}^{l}$ (Theorem 4). Our work is motivated by a result of Rosay [9] concerning the regularity at the edge of a holomorphic function in a wedge whose distribution boundary value is a continuous function on the edge. Another specific reason for this article is that we want to apply the main result, Theorem 1, in a forthcoming paper "Mappings of strongly pseudoconvex Cauchy-Riemann manifolds," and there does not seem to exist a precise reference, in spite of the fact that the question of boundary values of holomorphic functions has been one of the central subjects of complex analysis for some time. Most of the existing results concern the boundary behaviour of holomorphic functions on domains with smooth boundaries (see $[5,8,12]$ and the references therein). Another type of domains on which this question has been investigated to some extend are the wedges $W=\mathbb{R}^{l}+i \Gamma \subset \mathbb{C}^{l}$ with the linear totally real edge $\mathbb{R}^{l} \subset \mathbb{C}^{l}$, where $\Gamma \subset \mathbb{R}^{l}$ is an open cone with vertex at the origin. See Vladimirov [14], Rudin [10], Koranyi [6, 7], and Carmichael and Mitrovic [3]. We wish to point out that our methods are very similar to those used by Rosay in [9].

Let $n, l \in \mathbb{Z}_{+}$. In the space $\mathbb{C}^{n} \times \mathbb{C}^{l}$ we denote the coordinates by $(z, w)$, where $z \in \mathbb{C}^{n}, w=s+i t \in \mathbb{C}^{l}$, and $t=\left(t_{1}, \ldots, t_{l}\right)$. Let $M \subset \mathbb{C}^{n+l}$ be a smooth real manifold, defined in a neighborhood of the origin by

$$
w=s+i \varphi(z, \bar{z}, s),
$$

Received by the editors April 30, 1990.

1980 Mathematics Subject Classification (1985 Revision). Primary 32A40.

Research supported by the Max-Planck-Institut für Mathematik in Bonn. 
where $\varphi$ is a smooth mapping with values in $\mathbb{R}^{l}, \varphi(0)=0, d \varphi(0)=0$. Then $M$ is a generic Cauchy-Riemann (CR) manifold of CR dimension $n$ and of real codimension $l$.

If $\Gamma \subset \mathbb{R}^{l}$ is an open, connected, convex cone with vertex $0 \in \mathbb{R}^{l}$, we define the corresponding wedge $\mathscr{W}=\mathscr{W}(\Gamma) \subset \mathbb{C}^{n+l}$ with edge $M$ by

$$
\begin{aligned}
\mathscr{W}(\Gamma) & =\left\{(z, w) \in \mathbb{C}^{n+l}: \operatorname{Im} w-\varphi(z, \bar{z}, \operatorname{Re} w) \in \Gamma\right\} \\
& =\left\{(z, s+i \varphi(z, \bar{z}, s)+i t): z \in \mathbb{C}^{n}, s \in \mathbb{R}^{l}, t \in \Gamma\right\} .
\end{aligned}
$$

Throughout this paper we shall understand $M$ and $\mathscr{W}$ as germs of sets at the origin in $\mathbb{C}^{n+l}$.

Fix a point $p=\left(p_{1}, p_{2}\right) \in M, p_{1} \in \mathbb{C}^{n}, p_{2} \in \mathbb{C}^{l}$. A p-curve in $\mathscr{W}$ is a continuous curve $\Lambda(t) \in \mathscr{W}(0 \leq t<1)$ with $\lim _{t \rightarrow 1} \Lambda(t)=p$. Denote by $\lambda(t)$ the projection of $\Lambda(t)$ onto the plane $L_{p_{1}}=\left\{z=p_{1}\right\} \times \mathbb{C}^{l}$ in the direction of $T_{p}^{\mathbb{C}} M$, i.e., $\Lambda(t)-\lambda(t)$ is parallel to $T_{p}^{\mathbb{C}} M$ for all $0 \leq t<1$. Here, $T_{p}^{\mathbb{C}} M=T_{p} M \cap i T_{p} M$ is the maximal complex tangent space of $M$ at $p$, of complex dimension $n$.

Let $\mathscr{W}_{p_{1}}=L_{p_{1}} \cap \mathscr{W} ;$ this is a wedge in $L_{p_{1}}$ with the totally real generic edge $M \cap L_{p_{1}}$.

Definition. A $p$-curve $\Lambda(t)$ in $\mathscr{W}$ is called admissible if there is a $t_{0}<1$ such that

(a) $\lambda(t) \in \mathscr{W}_{p_{1}}\left(t_{0} \leq t<1\right)$, and $\lambda(t)$ is nontangential in $\mathscr{W}_{p_{1}}$ in the sense that

$$
|\lambda(t)-p| \leq A \cdot \operatorname{dist}\left(\lambda(t), \partial \mathscr{W}_{p_{1}}\right) \quad\left(t_{0} \leq t<1\right)
$$

for some $A<\infty$, and

$$
\lim _{t \rightarrow 1}|\Lambda(t)-\lambda(t)|^{2} / \operatorname{dist}\left(\lambda(t), \partial \mathscr{W}_{p_{1}}\right)=0 .
$$

A continuous function $f$ on $\mathscr{W}$ is said to have the admissible limit $B$ at $p \in M$ if

$$
\lim _{t \rightarrow 1} f(\Lambda(t))=B
$$

for every admissible $p$-curve $\Lambda(t)$ in $\mathscr{W}$.

Theorem 1. Let $M \subset \mathbb{C}^{n+l}$ be a Cauchy-Riemann manifold (1.1) of class $\mathscr{C}^{2}$, and let $\mathscr{W}$ be a wedge (1.2) with edge $M$. Then every bounded holomorphic function $f$ on $\mathscr{W}$ has an admissible limit $f^{*}(p)$ at almost every point $p \in M$ with respect to the surface measure on $M$.

Of course, the function $f^{*} \in L^{\infty}(M)$ is also the distribution boundary value of $f$ on $M$ in this case (see definition below).

Remark 1. This theorem is well known in the case $l=1$ when $M$ is a hypersurface in $\mathbb{C}^{n+1}$ (see [8 or 12]). Our terminology differs somewhat from the standard one in this case. Our notion of an admissible limit coincides with Rudin's restricted $K$-limit ( $K$ for Koranyi), see [12, p. 170]. On the other hand, Koranyi [7] used the term admissible limit for what is now usually called a $K$-limit (see [12, p. 76]). In this case one requires (1.3) along every $p$-curve $\Lambda(t)$ in $\mathscr{W}$ satisfying the weaker condition

$$
|\lambda(t)-p|<\alpha \cdot \operatorname{dist}(\Lambda(t), \partial \mathscr{W}) \quad(0 \leq t<1)
$$


for some $\alpha<\infty$. We do not know whether our Theorem 1 holds with admissible limits replaced by $K$-limits.

Remark 2. One can obtain better results concerning the convergence of $f$ in directions parallel to $T_{p}^{\mathbb{C}} M$ if one imposes suitable conditions on the geometry of $M$. As we shall see in the proof, the crucial property is the existence of sufficiently large complex discs in $\mathscr{W}$ in directions parallel to $T_{p}^{\mathbb{C}} M$ on which one can apply the Lindelöf-Chirka principle.

Remark 3. Although Fatou-type theorems are most interesting in the case of holomorphic functions, they can often be proved also for nonholomorphic functions whose $\bar{\partial}$-derivative does not grow too fast near the boundary. In the situation of Theorem 1 we suppose that $M$ is of class $\mathscr{C}^{k+2}$ for some $k \geq 0$, and $f \in \mathscr{C}^{1}(\mathscr{W})$ is a bounded function satisfying the estimates

$$
\begin{aligned}
& |\bar{\partial} f(z, w)|=\mathscr{O}\left(\operatorname{dist}((z, w), M)^{-1 / 2}\right), \\
& |\partial f(z, w)|=\mathscr{O}\left(\operatorname{dist}((z, w), M)^{-k-1}\right) .
\end{aligned}
$$

Then the conclusion of Theorem 1 holds for $f$. Here, as usual,

$$
\bar{\partial} f=\sum \frac{\partial f}{\partial \bar{z}_{j}} \cdot d \bar{z}_{j}+\sum \frac{\partial f}{\partial \bar{w}_{j}} \cdot d \bar{w}_{j}
$$

We indicate the proof of Theorem 1 under these weaker hypotheses at the end of $\S 2$ below.

For each $p \in M$ and $\alpha>1$ we define a nontangential approach region $\mathscr{D}_{p}^{\alpha} \subset \mathscr{W}$ by

$$
\mathscr{D}_{p}^{\alpha}=\{(z, w) \in \mathscr{W}:|(z, w)-p|<\alpha \cdot \operatorname{dist}((z, w), \partial \mathscr{W})\} .
$$

Of course $\mathscr{D}_{p}^{\alpha}$ may be empty if $\alpha$ is too small, depending on the size of the cone $\Gamma$ determining $\mathscr{W}$. However, as $\alpha \rightarrow \infty$, the regions $\mathscr{D}_{p}^{\alpha}$ increase to the entire wedge $\mathscr{W}$. A continuous function $f$ on $\mathscr{W}$ has the nontangential limit $B$ at $p \in M$ if

$$
\lim _{\mathscr{D}_{p}^{\alpha} \ni(z, w) \rightarrow p} f(z, w)=B
$$

for all (sufficiently large) $\alpha$.

A simple calculation shows that we can choose a sufficiently small neighborhood $U=U(p, \alpha)$ of $p$ in $\mathbb{C}^{n+l}$ such that every $p$-curve $\Lambda(t)$ in $\mathscr{D}_{p}^{\alpha} \cap U$ is admissible. Thus we have

Corollary 2. Under the hypotheses of Theorem 1, $f$ admits a nontangential limit $f^{*}(p)$ at almost every point $p \in M$.

Theorem 1 still holds if we only assume that the holomorphic function $f$ on $\mathscr{W}$ is bounded on every finer wedge $\mathscr{W}^{\prime}<\mathscr{W}$ in a neighborhood of $M$. (Recall that a cone $\Gamma^{\prime} \subset \mathbb{R}^{l}$ is finer than $\Gamma$ if $\Gamma^{\prime} \cap S$ is a relatively compact subset of $\Gamma \cap S$, where $S$ is the unit sphere in $\mathbb{R}^{l}$. Similarly, a wedge $\mathscr{W}^{\prime}=\mathscr{W}\left(\Gamma^{\prime}\right)$ of the form (1.2) is finer than $\mathscr{W}=\mathscr{W}(\Gamma)$ if $\Gamma^{\prime}$ is finer than $\Gamma$. We shall denote this by $\mathscr{W}^{\prime}<\mathscr{W}$.) Our proof will show that the last condition is satisfied if $f$ admits a distribution boundary value (bv $f$ ) that is a bounded function on $M$.

Denote by $d=d(z, w)$ the distance from a point $(z, w) \in \mathscr{W}$ to the edge $M$. (Clearly $d$ is proportional to $|\operatorname{Im} w-\varphi(z, \bar{z}, \operatorname{Re} w)|$.) Suppose that $M$ 
is of class $\mathscr{C}^{k+2}$ for some $K \in \mathbb{Z}_{+}$. If $f$ is a holomorphic function on $\mathscr{W}$ satisfying the growth condition

$$
|f(z, w)|=\mathscr{O}\left(d(z, w)^{-k}\right),
$$

then $f$ admits a distribution boundary value (bv $f$ ) on $M$ (Straube [13] and Rosay [9]), in the sense that for each test function $\chi \in \mathscr{C}_{0}^{k+1}\left(\mathbb{C}^{n+l}\right)$ supported in a small neighborhood of 0 we have

$$
\text { (bv } f, \chi)=\lim _{\Gamma^{\prime} \ni t \rightarrow 0} \int_{\mathbb{C}^{n} \times \mathbb{R}^{l}} \chi \cdot f(z, s+i \varphi(z, \bar{z}, s)+i t) d \nu(z, s),
$$

where $d \nu(z, s)$ is the Lebesgue measure on $\mathbb{C}^{n} \times \mathbb{R}^{l}$ and $\Gamma^{\prime}$ is any cone finer than $\Gamma$. The same holds if we replace $t \in \Gamma^{\prime}, t \rightarrow 0$ by a sequence of mappings $t_{j}(z, s) \in \Gamma^{\prime}$ that tend to zero in the $\mathscr{C}^{k+2}$ sense when $j \rightarrow \infty$.

Proposition 3. Suppose that $f \in \mathscr{O}(\mathscr{W})$ satisfies the growth condition (1.5). If (bv $f$ ) is (the integration against) a bounded measurable function on $M$, then $f$ is bounded on every finer wedge $\mathscr{W}^{\prime}<\mathscr{W}$ near $M$, and it has the admissible limit (bv $f)(p)$ at almost every point $p \in M$.

Remark. Another sufficient condition for a holomorphic function $f \in \mathscr{O}(\mathscr{W})$ to be bounded near the edge was found by Zav'yalov and Drozhzhinov [15], Khurumov [16], and Pinchuk and Hasanov [17]: If $f$ is bounded on a manifold $\widetilde{M} \subset \mathscr{W}$ of class $\mathscr{C}^{2}$ with boundary $M$ (so $\left.\operatorname{dim} \widetilde{M}=\operatorname{dim} M+1\right)$, then $f$ is bounded on every wedge $\mathscr{W}^{\prime}<\mathscr{W}$ near $M$.

When the function $f$ is bounded $(k=0)$, it follows immediately from the Banach-Alaoglu's theorem that bv $f$ is a bounded measurable function on $M$. Namely, given any sequence $t_{j} \in \Gamma^{\prime}, t_{j} \rightarrow 0$, the family of functions

$$
(z, s) \rightarrow f\left(z, s+i \varphi(z, \bar{z}, s)+i t_{j}\right)
$$

is bounded in $L^{\infty}=\left(L^{1}\right)^{*}$, so there is a subsequence converging to $f^{*} \in L^{\infty}$ in the weak- $*$ topology. Hence (1.6) holds for this sequence, with bv $f$ replaced by $f^{*}$. This proves that bv $f=f^{*} \in L^{\infty}$ as distributions on $M$.

A natural question is whether Theorem 1 holds in the case when (bv $f$ ) exists and is an integrable function on $M$, i.e., $($ bv $f) \in L^{1}(M)$. It seems that a positive answer is known only for the case when $M$ is hypersurface [8].

In this context we recall a result of Rosay [9]: If (bv $f$ ) is (the integration against) a continuous function on $M$, then $f$ extends continuously from every finer wedge $\mathscr{W}^{\prime}<\mathscr{W}$ to $M$, assuming the values (bv $f$ ) on $M$. See also the papers by Baouendi and Treves [1, 2].

To prove the announced results we use a standard method: we straighten the edge $M$ along the totally real submanifolds $M_{z}=\{z=$ const $\} \cap M$ and study the boundary behavior of nonholomorphic functions whose $\bar{\partial}_{w}$-derivatives satisfy good growth estimates near the linear edge $\mathbb{C}^{n} \times \mathbb{R}^{l}$. This will be explained in the following sections.

It is my pleasure to thank the Max-Planck-Institut für Mathematik in Bonn for its hospitality and support.

Remark added to the proof. Recently J. P. Rosay studied the boundary values of holomorphic functions in wedges in the article On the radial maximal function and the Hardy-Littlewood maximal function in wedges. 


\section{BOUNDARY VALUES OF FUNCTIONS WITH BOUNDED $\bar{\partial}$}

We use the same notation as Rosay in [9]. Let $N, l \in \mathbb{Z}_{+}$. In the space $\mathbb{R}^{N} \times \mathbb{C}^{l}$ we denote the coordinates by $(x, w), w=s+i t$. Let $\Gamma_{0}$ be the cone

$$
\Gamma_{0}=\left\{t=\left(t_{1}, \ldots, t_{l}\right) \in \mathbb{R}^{l}:\left|t_{j}\right|<t_{1}, 2 \leq j \leq l\right\},
$$

and let $\mathscr{W}_{0}$ be the corresponding wedge

$$
\mathscr{W}_{0}=\mathbb{R}^{N} \times\left(\mathbb{R}^{l}+i \Gamma_{0}\right)=\left\{(x, s+i t): x \in \mathbb{R}^{N}, s \in \mathbb{R}^{l}, t \in \Gamma_{0}\right\} .
$$

The results announced in $\S 1$ will follow from

Theorem 4. Let $g(x, w)$ be a continuously differentiable function on $\mathscr{W}_{0}$ satisfying

$$
|g|=\mathscr{O}\left(t_{1}^{-k}\right), \quad\left|\partial g / \partial \bar{w}_{j}\right|=\mathscr{O}\left(t_{1}^{-1+\varepsilon}\right), \quad 1 \leq j \leq l,
$$

for some $k \in \mathbb{Z}_{+}$and $\varepsilon>0$. If the distribution boundary value (bv $g$ ) on $\mathbb{R}^{N} \times \mathbb{R}^{l}$ (that exists according to Rosay [9]) is a bounded measurable function, then $g$ is bounded on $\mathscr{W}_{0}$ near $\mathbb{R}^{N} \times \mathbb{R}^{l}$, and there is a set $F \subset \mathbb{R}^{N} \times \mathbb{R}^{l}$ of full measure so that at each point $(x, s) \in F$, the restriction $g(x, \cdot)$ to the wedge $\{x\} \times\left(\mathbb{R}^{l}+i \Gamma_{0}\right)$ has the nontangential limit (bv $\left.g\right)(x, s)$.

Remark. Contrary to the first appearance this result is completely local since the growth condition on $\bar{\partial}_{w} g$ is preserved if we multiply $g$ by a smooth cutoff function $\psi \in \mathscr{C}_{0}^{\infty}\left(\mathbb{R}^{N} \times \mathbb{C}^{l}\right)$ whose derivatives $\partial \psi / \partial \bar{w}_{j}$ vanish to infinite order on $\mathbb{R}^{N} \times \mathbb{R}^{l}(1 \leq j \leq l)$.

If $g$ is bounded on $\mathscr{W}_{0}$, the boundary value (bv $g$ ) exists and is in $L^{\infty}\left(\mathbb{R}^{N} \times \mathbb{R}^{l}\right)$. (See the remark following Proposition 3 above.) Thus we have

Corollary 5. If $g$ is a bounded $\mathscr{C}^{1}$ function in $\mathscr{W}_{0}$ satisfying (2.1), then at almost every point $(x, s) \in \mathbb{R}^{N} \times \mathbb{R}^{l}, g(x, \cdot)$ has a nontangential limit at $(x, s)$ within the wedge $\{x\} \times\left(\mathbb{R}^{l}+i \Gamma_{0}\right)$.

In these results the variable $x$ is merely a parameter, as we have no assumptions and conclusions on the nontangential behavior of $g$ in the $x$-direction.

Remark. Our condition (2.1) concerning the growth of $\bar{\partial}_{w} g$ near the edge is not the best one possible, but it is easy to verify and convenient to use. For functions of one complex variable there is a sharper result, due to Nagel and Rudin [12, p. 235]: If $g$ is a bounded $\mathscr{C}^{1}$ function in a rectangle $Q=(a, b) \times(0, c) \subset$ $\mathbb{R}^{2}=\mathbb{C}$ such that $\partial g / \partial \bar{w} \in L^{p}(Q)$ for some $p>1$, then $\lim _{y \rightarrow 0} g(x+i y)$ exists for almost all $x \in(a, b)$.

However, in most applications the condition (2.1) is sufficient. In the proof of Theorem 1 we shall only need that $\bar{\partial}_{w} g$ is bounded. In the proof of the result by Nagel, Rudin, and Wainger [12, p. 238] on nontangential boundary values of a function $f \in H^{\infty}(\mathscr{D})$ along a $\mathscr{C}^{1}$ curve in the boundary of $\mathscr{D}$ that is everywhere transverse to the complex tangent space $T_{p}^{\mathbb{C}} \partial \mathscr{D}$ one obtains a function $g$ on $Q$ as above such that $|\partial g / \partial \bar{w}|=\mathscr{O}\left((\operatorname{Im} w)^{-1 / 2}\right)$.

We will first show how the results of $\S 1$ follow from Theorem 4 . The proof of Theorem 4 will be given in $\S 3$ below.

Let $N=2 n, \mathbb{R}^{2 n}=\mathbb{C}^{n}$. Suppose that the manifold $M(1.1)$ is of class $\mathscr{C}^{k+2}$. We extend the mapping $\varphi$ to a $\mathscr{C}^{k+2}$ map in a neighborhood of the 
origin in $\mathbb{C}^{n} \times \mathbb{C}^{l}$ so that $\bar{\partial}_{w} \varphi$ and its derivatives of order $\leq k+1$ vanish on $\mathbb{C}^{n} \times \mathbb{R}^{l}$. The extended map

$$
\Phi(z, w)=(z, w+i \varphi(z, w))=(z, \zeta(z, w))
$$

is a local $\mathscr{C}^{k+2}$ diffeomorphism near the origin that maps $\mathbb{C}^{n} \times \mathbb{R}^{l}$ onto $M$.

Let $f$ be a holomorhic function on $\mathscr{W}$ satisfying the hypothesis of Proposition 3. We must show that the restriction of $f$ to any finer wedge $\mathscr{W}^{\prime}<\mathscr{W}$ is bounded near $M$ and has the admissible limit (bv $f)(p)$ at almost every point $p \in M$.

Choose a wedge $\mathscr{W}^{\prime \prime}$ satisfying $\mathscr{W}^{\prime}<\mathscr{W}^{\prime \prime}<\mathscr{W}$. The inverse images $\Phi^{-1}\left(\mathscr{W}^{\prime}\right) \subset \Phi^{-1}\left(\mathscr{W}^{\prime \prime}\right) \subset \Phi^{-1}(\mathscr{W})$ are wedge-like domains near the origin, with the edge $\mathbb{C}^{n} \times \mathbb{R}^{l}$. We can find a finite number of cones $\Gamma_{1}, \Gamma_{2}, \ldots, \Gamma_{\nu} \subset \mathbb{R}^{l}$ isomorphic to the cone $\Gamma_{0}$ so that the corresponding wedges $\mathscr{W}_{j}=\mathbb{C}^{n} \times\left(\mathbb{R}^{l}+i \Gamma_{j}\right)$ satisfy the following inclusions in a sufficiently small neighborhood of the origin:

$$
\Phi^{-1}\left(\mathscr{W}^{\prime}\right) \subset \bigcup_{j=1}^{\nu} \mathscr{W}_{j} \subset \Phi^{-1}\left(\mathscr{W}^{\prime \prime}\right) .
$$

Let $g=f \circ \Phi \in \mathscr{C}^{k+2}\left(\Phi^{-1}(\mathscr{W})\right)$. Then $|g|=\mathscr{O}\left(d^{-k}\right)$ on each wedge $\mathscr{W}_{j}$, where $d$ denotes the distance from the wedge. The chain rule gives

$$
\partial g / \partial \bar{w}_{j}=\sum_{s=1}^{l} \partial f / \partial \zeta_{s} \cdot \partial \zeta_{s} / \partial \bar{w}_{j}
$$

Since $|f|=\mathscr{O}\left(d^{-k}\right)$ on $\mathscr{W}$, the Cauchy estimates imply $\left|\partial f / \partial \zeta_{s}\right|=\mathscr{O}\left(d^{-k-1}\right)$ on $\mathscr{W}^{\prime \prime}$ for $1 \leq s \leq l$. We also have $\left|\partial \zeta_{s} / \partial \bar{w}_{j}\right|=\mathscr{O}\left(d^{k+1}\right)$ by the construction of $\Phi$, so it follows that $\partial g / \partial \bar{w}_{j}$ is bounded on $\mathscr{W}_{s}$ for $1 \leq j \leq l$. Since the distribution boundary value (bv $g$ ) (that exists by [9]) is the pull-back of (bv $f$ ) by $\Phi$, we conclude that (bv $g) \in L^{\infty}\left(\mathbb{C}^{n} \times \mathbb{R}^{l}\right)$. Theorem 4 now implies that $g$ is bounded on each $\mathscr{W}_{s}$ near $\mathbb{C}^{n} \times \mathbb{R}^{l}$, so $f$ is bounded on $\mathscr{W}^{\prime}$ near $M$.

The second part of Theorem 4 implies that for almost every point $p_{0}$ $=\left(z_{0}, s_{0}\right) \in \mathbb{C}^{n} \times \mathbb{R}^{l}$, the restriction $g\left(z_{0}, \cdot\right)$ has the nontangential limit (bv $g)\left(z_{0}, s_{0}\right)$ within the wedge $\left\{z_{0}\right\} \times\left(\mathbb{R}^{l}+i \Gamma_{s}\right)$ at $\left(z_{0}, s_{0}\right)$. Going back to $f$, this says that $f\left(z_{0}, \cdot\right)$ has the nontangential limit (bv $\left.f\right)\left(z_{0}, \zeta_{0}\right)$ at $\left(z_{0}, \zeta_{0}\right)=\Phi\left(z_{0}, s_{0}\right)$ within the wedge $\left\{z=z_{0}\right\} \cap \mathscr{W}^{\prime}$. This holds for every $\mathscr{W}^{\prime}<\mathscr{W}$, so the same is true for $\mathscr{W}$.

It remains to show that $f$ has the same admissible limit within the entire wedge $\mathscr{W}$ at $p_{0}=\left(z_{0}, \zeta_{0}\right)$. This follows by applying the Lindelöf-Chirka principle [12, p. 168]. We shall explain briefly the idea.

Let $\Lambda(t) \in \mathscr{W}$ be an admissible $p_{0}$-curve, and let $\lambda(t)$ be its projection onto $\left\{z=z_{0}\right\} \times \mathbb{C}^{l}$ so that $\Lambda(t)-\lambda(t)$ is parallel to $T_{p_{0}}^{\mathbb{C}} M$. Denote by $R(t)$ the radius of the largest disc in $\mathbb{C}$ that is mapped into $\mathscr{W}$ by the mapping

$$
\mathbb{C} \ni \zeta \rightarrow(1-\zeta) \cdot \lambda(t)+\zeta \cdot \Lambda(t) \in \mathbb{C}^{n+l} .
$$

Since $\Lambda$ is admissible, a simple calculation shows that $R(t) \rightarrow \infty$ as $t \rightarrow 1$.

The function $g(\zeta)=f((1-\zeta) \lambda(t)+\zeta \Lambda(t))$ is bounded holomorphic on $|\zeta|<R(t)$, with $\|g\|_{\infty} \leq\|f\|_{\infty}$. The Schwarz lemma implies

$$
|f(\Lambda(t))-f(\lambda(t))|=|g(1)-g(0)| \leq 2\|f\|_{\infty} / R(t) .
$$


When $t \rightarrow 1, f(\lambda(t)) \rightarrow($ bv $f)\left(p_{0}\right)$ since the curve $\lambda(t)$ approaches $p_{0}$ nontangentially within the wedge $\left\{z=z_{0}\right\} \cap \mathscr{W}$. Since the right-hand side goes to zero, we have $f(\Lambda(t)) \rightarrow(\mathrm{bv} f)\left(p_{0}\right)$ as well. Thus Theorem 1 and Proposition 3 are proved, provided that Theorem 4 holds.

We shall now indicate the proof of Theorem 1 under the weaker assumptions (1.4) on $f$. Let $g=f \circ \Phi$ be as above. By the chain rule,

$$
\begin{aligned}
\partial g / \partial \bar{w}_{j} & =\sum_{s=1}^{l} \partial f / \partial \zeta_{s} \cdot \partial \zeta_{s} / \partial \bar{w}_{j}+\sum_{s=1}^{l} \partial f / \partial \bar{\zeta}_{s} \cdot \partial \bar{\zeta}_{s} / \partial \bar{w}_{j} \\
& =\mathscr{O}\left(d^{-k-1}\right) \cdot \mathscr{O}\left(d^{k+1}\right)+\mathscr{O}\left(d^{-1 / 2}\right) \cdot \mathscr{O}(1)=\mathscr{O}\left(d^{-1 / 2}\right),
\end{aligned}
$$

so $g$ still satisfies Theorem 4 . The only other place where we used the fact that $f$ was bounded holomorphic was in the Lindelöf-Chirka principle. In the nonholomorphic case we shall require the following lemma.

Lemma 6. Denote by $\Delta=\{|\zeta|<1\}$ the unit disc in $\mathbb{C}$. For each compact subset $K \subset \Delta$ and for each $\alpha \in(0,1)$ there is a constant $C=C(K, \alpha)$ such that every function $f \in \mathscr{C}^{1}(\bar{\Delta})$ satisfies the estimate

$$
\left|f\left(z_{1}\right)-f\left(z_{2}\right)\right| \leq C\left(\|f\|_{\infty}+\|\partial f / \partial \bar{z}\|_{\infty}\right) \cdot\left|z_{1}-z_{2}\right|^{\alpha}, \quad z_{1}, z_{2} \in K
$$

Here, $\left.\|f\|_{\infty}=\sup \{|f(z)|): z \in \bar{\Delta}\right\}$.

Proof. By the Cauchy formula we have

$$
\begin{aligned}
f(z) & =\frac{1}{2 \pi i} \oint_{|\zeta|=1} \frac{f(\zeta)}{\zeta-z} d \zeta-\frac{1}{2 \pi i} \iint_{|\zeta| \leq 1} \frac{(\partial f / \partial \bar{\zeta})(\zeta)}{\zeta-z} d \bar{\zeta} \wedge d \zeta \\
& =\Phi(f)(z)+T(\partial f / \partial \bar{\zeta})(z), \quad z \in \Delta .
\end{aligned}
$$

The operator $T$ maps $L^{\infty}(\Delta)$ boundedly into the Hölder space $\mathscr{C}^{\alpha}(\Delta)$ [18, p. 34], so $T(\partial f / \partial \bar{\zeta})$ satisfies the estimate (2.3) with a constant $C_{1}<\infty$ depending only on $\alpha$. Also, since $\Phi(f)$ is holomorphic, $\|\Phi(f)\|_{\infty} \leq\|f\|_{\infty}$, and the Cauchy estimates imply

$$
\left|\Phi(f)\left(z_{1}\right)-\Phi(f)\left(z_{2}\right)\right| \leq C_{2} \cdot\|f\|_{\infty} \cdot\left|z_{1}-z_{2}\right|,
$$

where $C_{2}$ depends on $K \subset \subset \Delta$. Combining the two estimates we obtain (2.3). Lemma 6 is proved.

Consider now the complex line $L_{t} \subset \mathbb{C}^{n+l}$ given by (2.2). Fix a $t<1$ and let $\varepsilon$ be the distance of $\lambda(t)$ to $M$. Within $L_{t}$ there is a disc $\Delta_{t} \subset \mathscr{W}$, centered at $\lambda(t)$, of radius comparable to $\varepsilon^{1 / 2}$, so that $\operatorname{dist}(p, M) \geq c \cdot \varepsilon$ for some $c>0$ and for all $p \in \Delta_{t}$. By our assumption on $f$ we have $|\bar{\partial} f| \lesssim \varepsilon^{-1 / 2}$ on $\Delta_{t}$. If we rescale $\Delta_{t}$ to the unit disc $\Delta=\{\eta \in \mathbb{C}:|\eta|<1\}$ so that $\gamma(t)$ corresponds to $\eta=0$ and denote by $h_{t}(\eta)$ the restriction of $f$ to $\Delta_{t}$ in the $\eta$-coordinate, then $\left\|\bar{\partial} h_{t}\right\|_{\infty} \leq C<\infty$, where $C$ is independent of $t$. Also, $\left\|h_{t}\right\|_{\infty} \leq\|f\|_{\infty}$. The condition that the curve $\Lambda(t)$ is admissible implies that the point $\eta(t) \in \Delta$ corresponding to $\Lambda(t) \in \Delta_{t}$ tends to zero as $t \rightarrow 1$, so Lemma 6 implies

$$
|f(\Lambda(t))-f(\lambda(t))|=\left|h_{t}(\eta(t))-h_{t}(0)\right| \rightarrow 0
$$


as $t \rightarrow 1$. This completes the proof of Theorem 1 , provided that Theorem 4 holds.

\section{Proof of Theorem 4}

Multiplying $g$ by a suitable cut-off function $\chi$ such that $\bar{\partial}_{w} \chi$ is flat on $\mathbb{R}^{N} \times \mathbb{R}^{l}$ we may assume that the support of $g$ is contained in $\mathscr{W}_{0} \cap\left\{|x|^{2}+|w|^{2}\right.$ $<1\}$. Let (bv $g$ ) $\in L^{\infty}\left(\mathbb{R}^{N} \times \mathbb{R}^{l}\right)$ be the distribution boundary value of $g$.

We treat first the one-variable situation. Let $\Pi_{+}=\{w=s+i t \in \mathbb{C}: t>0\}$, and let $h \in \mathscr{C}^{1}\left(\bar{\Pi}_{+}\right)$have support contained in $\{|w|<1\}$. The formula of Stokes gives the following Cauchy's formula with weight:

$$
h(i)=\frac{1}{\pi} \int_{-\infty}^{\infty} \frac{h(\tau)}{\tau^{2}+1} d \tau-\frac{1}{\pi} \int_{\Pi^{+}} \partial h / \partial \bar{\zeta} \cdot \frac{1}{\zeta^{2}+1} \cdot d \bar{\zeta} \wedge d \zeta .
$$

Replacing $h(\zeta)$ by $h(s+t \zeta)$ for a fixed pair $s \in \mathbb{R}, t>0, s^{2}+t^{2}<1$, we obtain

$$
h(s+i t)=\frac{1}{\pi} \int_{-\infty}^{\infty} \frac{h(s+t \tau) d \tau}{\tau^{2}+1}-\frac{t}{\pi} \int_{\Pi^{+}} \partial h / \partial \bar{\zeta}(s+t \zeta) \frac{1}{\zeta^{2}+1} d \bar{\zeta} \wedge d \zeta
$$

Suppose now that $h$ is a $\mathscr{C}^{1}$ function in $\Pi_{+}$with bounded support, but it is not $\mathscr{C}^{1}$ up to the boundary $\partial \Pi_{+}=\mathbb{R}$. If we assume that $|\partial h / \partial \bar{\zeta}|=\mathscr{O}\left((\operatorname{Im} \zeta)^{-1+\varepsilon}\right)$ for some $\varepsilon>0$ and that $h$ has a distribution boundary value (bv $h) \in L^{\infty}(\mathbb{R})$, then the formula (3.1) still holds, provided that we replace $h$ by (bv $h$ ) in the first integral. This can be seen by applying our formula to the translates $h_{\eta}(\zeta)=h(\zeta+i \eta)$ for $\eta>0$ and letting $\eta \rightarrow 0$. The first integral converges by definition since $1 /\left(\tau^{2}+1\right)$ is a test function (recall that $h$ has bounded support!). In the second integral we have

$$
\left|\left(\partial h_{\eta} / \partial \bar{\zeta}\right)(s+t \zeta)\right| \leq C \cdot(t \operatorname{Im} \zeta)^{-1+\varepsilon},
$$

and we integrate over the set

$$
\left\{\zeta \in \Pi_{+}:|s+t \zeta|<1\right\} \subset\left\{\zeta \in \Pi_{+}:|\zeta|<2 / t\right\}
$$

Thus the second term in (3.1) is bourided up to a constant factor by

$$
t^{\varepsilon} \cdot \int_{\substack{|\zeta|<2 / t \\ y>0}} y^{-1+\varepsilon} \frac{1}{\left|\zeta^{2}+1\right|} d x d y, \quad \zeta=x+i y,
$$

that can be estimated from above by

$$
t^{\varepsilon}(\log (2 / t)+\mathscr{O}(1))=t^{\varepsilon} \log 1 / t+\mathscr{O}\left(t^{\varepsilon}\right) .
$$

The dominated convergence theorem applies, and we have (3.1).

As $T \searrow 0$, the above estimate shows that the second integral converges to zero, uniformly for $s \in \mathbb{R}$. Thus $h$ is bounded on $\Pi_{+}$and has a nontangential limit at $s \in \mathbb{R}$ precisely when the first integral in (3.1) has these properties. Replacing $s+t \tau$ by $\tau$ we get the integral

$$
\tilde{h}(s+i t)=\frac{1}{\pi} \int_{-\infty}^{\infty} h(\tau) \frac{t}{(\tau-s)^{2}+t^{2}} \cdot d \tau .
$$

This is just the Poisson integral $P[h]$ of $h$ in $\Pi_{+}$. From the classical theory [5] we know that for all $h \in L_{\infty}(\mathbb{R}), P[h]$ is bounded and has nontangential 
limit $h(s)$ at almost every $s \in \mathbb{R}$; certainly this is true at every Lebesgue point of $h$. This proves Theorem 4 in the case $N=0, l=1$.

Recall that a point $x_{0} \in \mathbb{R}^{m}$ is a Lebesgue point of a locally integrable function $h \in L_{\text {loc }}^{1}\left(\mathbb{R}^{m}\right)$ if

$$
\lim _{r \searrow 0} \frac{1}{\nu\left(B\left(x_{0}, r\right)\right)} \cdot \int_{B\left(x_{0}, r\right)}\left|h(x)-h\left(x_{0}\right)\right| d \nu(x)=0,
$$

where $\nu$ is the Lebesgue measure on $\mathbb{R}^{m}$ and $B\left(x_{0}, r\right)=\left\{\left|x-x_{0}\right|<r\right\}$. The classical Lebesgue's differentiation theorem asserts that the set of Lebesgue points of every $h \in L_{\text {loc }}^{1}\left(\mathbb{R}^{m}\right)$ has full measure [4, p. 93].

We now proceed to the proof of Theorem 4 in the general situation. For each vector $t \in \Gamma_{0} \backslash\{0\}$ and for each point $(x, s) \in \mathbb{R}^{N} \times \mathbb{R}^{l}$ we have the complex half-plane

$$
\Pi_{t}^{+}(x, s)=\left\{(x, s+t \zeta) \in \mathbb{R}^{N} \times \mathbb{C}^{l}: \operatorname{Im} \zeta>0\right\} \subset \mathscr{W}_{0}
$$

with boundary $l_{t}(x, s) \subset \mathbb{R}^{N} \times \mathbb{R}^{l}$.

A priori we do not know that the restriction of $g$ to $\Pi_{t}^{+}(x, s)$ has the distribution boundary value on $l_{t}(x, s)$ equal to $(\mathrm{bv} g)$, restricted to $l_{t}(x, s)$. To avoid this difficulty we use regularization as in [11]. Choose a function $\chi \in \mathscr{C}_{0}^{\infty}\left(\mathbb{R}^{N} \times \mathbb{R}^{l}\right), \chi \geq 0$, with $\int \chi d x d s=1$, and let $\chi_{\delta}=\chi(\cdot / \delta) / \delta^{N+l}$ for $\delta>0$. Let $g_{\delta}=g * \chi_{\delta}$ be the convolution of $g$ with $\chi_{\delta}$ in the variables $(x, s)$ :

$$
g_{\delta}(x, s+i t)=\int g\left(x^{\prime}, s^{\prime}+i t\right) \chi_{\delta}\left(x-x^{\prime}, s-s^{\prime}\right) d x^{\prime} d s^{\prime} .
$$

Clearly $g_{\delta}$ satisfies the same growth conditions as $g$, uniformly with respect to $\delta$. Also, $g_{\delta}$ is continuous up to the edge $\mathbb{R}^{N} \times \mathbb{R}^{l}$ and equals (bv $g$ ) $* \chi_{\delta}$ there. As $\delta$ goes to $0,(\mathrm{bv} g) * \chi_{\delta}(x, s)$ converges boundedly to (bv $\left.g\right)(x, s)$ at every Lebesgue point $(x, s)$ of (bv $g$ ).

Let $E \subset \mathbb{R}^{N} \times \mathbb{R}^{l}$ denote the set of Lebesgue points of (bv $g$ ). Fubini's theorem implies that for each $t \in \Gamma_{0} \backslash\{0\}$ there is a set $E_{t} \subset E$ of full Lebesgue measure such that for every $(x, s) \in E_{t}$; the intersection $E \cap l_{t}(x, s)$ is a set of full one-dimensional Lebesgue measure in $l_{t}(x, s)$. For any such point $(x, s) \in E_{t}$ we apply the formula (3.1) to the function $g_{\delta}$, restricted to $\Pi_{t}^{+}(x, s)$ :

$$
\begin{aligned}
g_{\delta}(x, s+i t)= & \frac{1}{\pi} \int_{-\infty}^{\infty} \frac{g_{\delta}(x, s+t \tau)}{\tau^{2}+1} \cdot d \tau \\
& -\frac{1}{\pi} \sum_{j=1}^{l} t_{j} \int_{\Pi_{+}} \frac{\partial g_{\delta}}{\partial \bar{w}_{j}}(x, s+t \zeta) \cdot \frac{1}{\zeta^{2}+1} \cdot d \bar{\zeta} \wedge d \zeta .
\end{aligned}
$$

Now let $\delta \searrow 0$. By the dominated convergence the second integral converges to the same expression with $g_{\delta}$ replace by $g$. In the first integral, $g_{\delta} \rightarrow$ bv $g$ boundedly almost everywhere on the line $l_{t}(x, s)$ (i.e., at each Lebesgue point of bv $g$ ), so the dominated convergence applies again. Thus

$$
\begin{aligned}
g(x, s+i t)= & \frac{1}{\pi} \int_{-\infty}^{\infty}(\text { bv } g)(x, s+t \tau) \frac{1}{\tau^{2}+1} d \tau \\
& -\frac{1}{\pi} \sum_{j=1}^{l} t_{j} \int_{\Pi^{+}} \frac{\partial g}{\partial \bar{w}_{j}}(x, s+t \zeta) \frac{1}{\zeta^{2}+1} d \bar{\zeta} \wedge d \zeta .
\end{aligned}
$$


Since $\left|t_{j}\right|<t_{1}$ on $\Gamma_{0}$, we can estimate the second integral by $C \cdot t_{1}^{\varepsilon} \cdot\left(\log 1 / t_{1}+\right.$ $\mathscr{O}(1))$ as in the one-variable case $\left(C\right.$ only depends on $g$ ). Since bv $g \in L^{\infty}$, this formula shows that $g$ is bounded on $\Pi_{t}^{+}(x, s)$ and the bound is independent of $(t, x, s)$, provided that $l_{t}(x, s) \cap E$ has full measure in $l_{t}(x, s)$. The union of the corresponding half-planes $\Pi_{t}^{+}(x, s)$ is everywhere dense in $\mathscr{W}_{0}$, so $g$ is bounded on $\mathscr{W}_{0}$. This proves the first assertion of Theorem 4 .

To prove the second assertion concerning the nontangential boundary values we choose a countable dense set of vectors $\left\{t_{j}\right\}_{j=1}^{\infty}$ in $\Gamma_{0} \backslash\{0\}$ and let $E_{t_{j}}=E_{j}$ be the corresponding sets of full measure in $E$. For each $j$ we let $F_{j}$ be the set of all points $(x, s) \in E_{j}$ with the property that $(x, s)$ is a Lebesgue point of the restriction of ( $\mathrm{bv} g$ ) to $l_{t_{j}}(x, s)$. This also is a set of full measure in $\mathbb{R}^{N} \times \mathbb{R}^{l}$. Finally let $F=\bigcap_{j=1}^{\infty} F_{j}$. Theorem 4 follows from

Lemma 7. At each point $(x, s) \in F$, the function $g(x, \cdot)$ has the nontangential limit (bv $g)(x, s)$ within the wedge $\{x\} \times\left(\mathbb{R}^{l}+i \Gamma_{0}\right) \subset\{x\} \times \mathbb{C}^{l}$.

Proof. Fix $(x, s) \in F$. By the construction of $F$ and the corresponding onevariable result we know that

$$
\lim _{\varepsilon \searrow 0} g\left(x, s+i \varepsilon t_{j}\right)=(\operatorname{bv} g)(x, s)
$$

for all $j \in \mathbb{Z}_{+}$. Our goal is to show that for every conical approach region $\Lambda \subset\left(\mathbb{R}^{l}+i \Gamma_{0}\right)$ with vertex at $(x, s)$ we have

$$
\lim _{\Lambda \ni w \rightarrow s} g(x, w)=(\text { bv } g)(x, s) .
$$

Without loss of generality we may take $(x, s)$ to be the origin in $\mathbb{R}^{N} \times \mathbb{C}^{l}$ and (bv $g)(0,0)=0$. For every sequence $\varepsilon_{j}>0, \varepsilon_{j} \searrow 0$, we consider the family of functions $h_{j}(\zeta)=g\left(0, \varepsilon_{j} \zeta\right), \zeta \in \Lambda$. The sequence is uniformly bounded on $\Lambda$. Moreover, the growth condition $\left|\bar{\partial}_{w} g\right|=\mathscr{O}\left(t_{1}^{-1+\varepsilon}\right)$ shows that on every compact subset $K \subset \Lambda$ we have

$$
\begin{aligned}
\partial h_{j} / \partial \bar{\zeta}_{k}(\zeta) & =\varepsilon_{j} \partial g / \partial \bar{w}_{k}\left(0, \varepsilon_{j} \zeta\right)=\varepsilon_{j} \mathscr{O}\left(\left(\varepsilon_{j} \operatorname{Im} \zeta\right)^{-1+\varepsilon}\right) \\
& =\mathscr{O}\left(\varepsilon_{j}^{\varepsilon}\right) \rightarrow 0 \quad \text { as } j \rightarrow \infty
\end{aligned}
$$

Lemma 6 implies that $\left\{h_{j}\right\}$ is uniformly Hölder continuous on every $K \subset \subset$ $\Lambda$, so there is a subsequence converging to a continuous function $h \in \mathscr{C}(\Lambda)$. The above estimate of $\left|\bar{\partial} h_{j}\right|$ implies $\bar{\partial} h=0$ (this is verified immediately in the sense of distributions), so the limit function $h$ is holomorphic in $\Lambda$.

Now (3.2) shows that $h$ equals (bv $g)(0,0)=0$ on each ray $\left\{i \lambda t_{j}: \lambda>0\right\}$. Since the union of these rays is dense in the cone $i \Gamma_{0}$, it follows by continuity that $h \equiv 0$ on the totally real submanifold $i \Gamma_{0} \cap \Lambda$ of $\Lambda \subset \mathbb{C}^{l}$ of maximal dimension $l$. Since $h$ is holomorphic, we conclude that $h \equiv 0$ in $\Lambda$. This holds for every sequence $\left\{\varepsilon_{j}\right\}$ converging to zero, so the usual argument by contradiction concludes the proof of Lemma 7.

\section{REFERENCES}

1. M. S. Baouendi and F. Trèves, A property of functions and distributions annihilated by a locally integrable system of complex vector fields, Ann. of Math. 113 (1981), 387-421.

2. M. S. Baouendi, H. Jacobowitz and F. Trèves, On the analyticity of C.R. mappings, Ann. of Math. 122 (1985), 365-400. 
3. R. D. Carmichael and D. Mitrovic, Distributions and analytic functions, Pitman Research Notes in Math., 206, Pitman, New York, 1989.

4. J. B. Folland, Real analysis, Wiley, New York, 1984.

5. J. B. Garnett, Bounded analytic functions, Academic Press, New York, 1981.

6. A. Koranyi, The Poisson integral for generalized half-planes and bounded symmetric domains, Ann. of Math. 82 (1965), 332-350.

7. _ Harmonic functions on Hermitian hyperbolic spaces, Trans. Amer. Math. Soc. 135 (1969), 507-516.

8. St. G. Krantz, Function theory of several complex variables, Wiley, New York, 1982.

9. J. P. Rosay, A propos de "wedges" et d' "edges", et de prolongements holomorphes, Trans. Amer. Math. Soc. 297 (1986), 63-72.

10. W. Rudin, Function theory in polydiscs, Benjamin, New York, 1969.

11. _ Lectures on the edge of the wedge theorem, CBMS Regional Conf. Ser. in Math., no. 6, Amer. Math. Soc., Providence, R.I., 1971.

12. _ Function theory in the unit ball of $\mathbb{C}^{n}$, Springer, New York, 1980.

13. E. Straube, Harmonic and analytic functions admitting a distribution boundary value, Ann. Scuola Norm. Sup. Pisa Cl. Sci. (4) 11 (1984), 559-591.

14. V. Vladimirov, Methods of the theory of functions of many complex variables, M.I.T. Press, Boston, Mass., 1966.

15. B. I. Zav'yalov and Yu. N. Drozhzhinov, On a multidimensional analogue of Lindelöf's theorem, Dokl. Akad. Nauk SSSR 262 (1982), 269-270; English transl., Soviet Math. Dokl. 25 (1982).

16. Yu. V. Khurumov, On Lindelöf's theorem in $\mathbb{C}^{n}$, Dokl. Akad. Nauk SSSR 273 (1983), 1325-1328; English transl., Soviet Math. Dokl. 28 (1983).

17. S. I. Pinchuk and S. V. Khasanov, Asymptotically holomorphic functions and their applications, Mat. Sb. 134 (176) (1987), 546-555; English transl., Math. USSR-Sb. 62 (1989), no. 2, 541-550.

18. I. N. Vekua, Verallgemeinerte analytische Funktionen, Akademie-Verlag, Berlin, 1963.

Max-Planck-Institut für Mathematik, Bonn, Germany

Current address: University of Wisconsin, Department of Mathematics, Madison, Wisconsin 53706

E-mail address: forstner@math.wisc.edu 